This item was submitted to Loughborough's Institutional Repository (https://dspace.lboro.ac.uk/) by the author and is made available under the following Creative Commons Licence conditions.

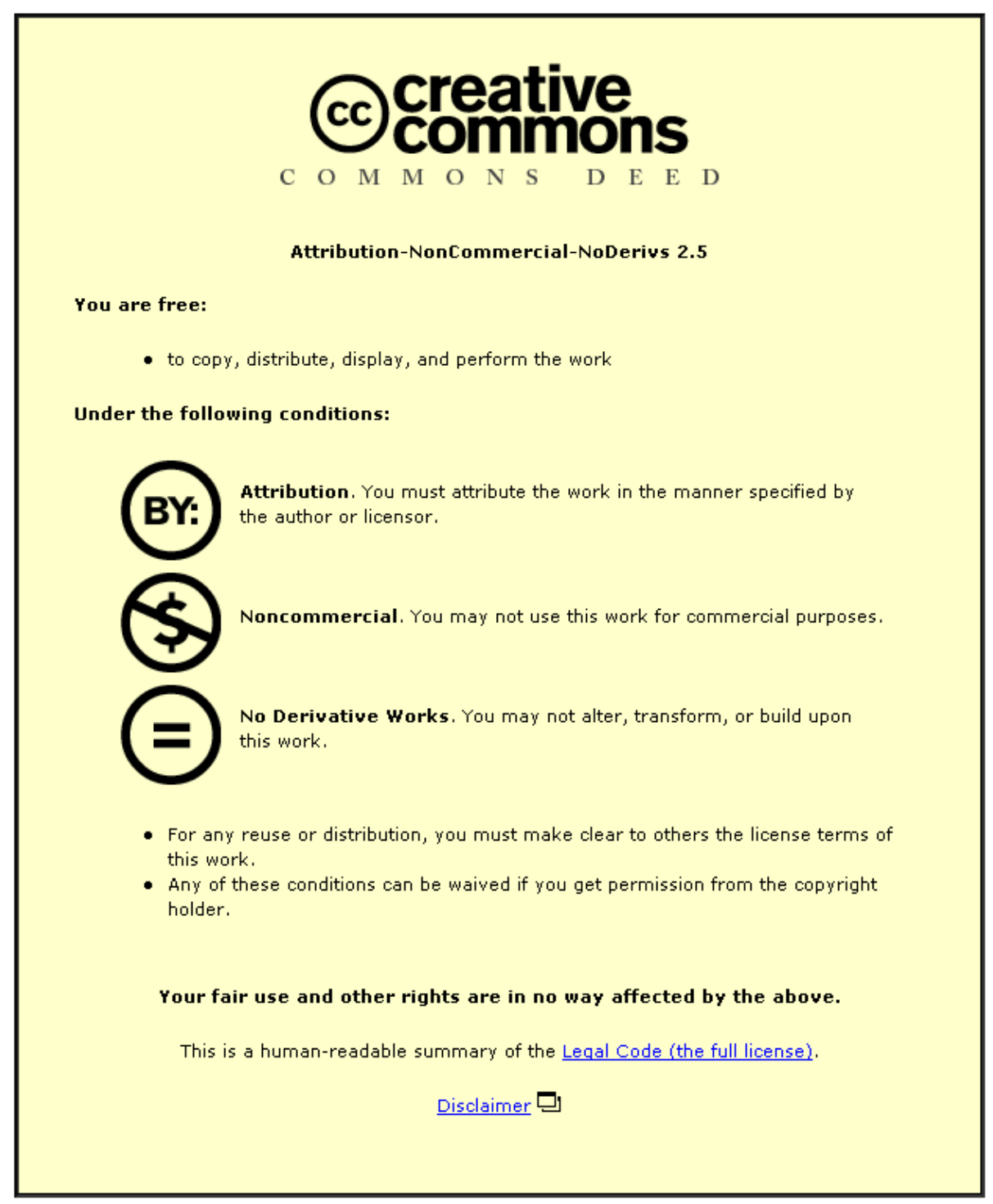

For the full text of this licence, please go to: http://creativecommons.org/licenses/by-nc-nd/2.5/ 


\title{
Gel casting of aqueous suspensions of $\mathrm{BaTiO}_{3}$ nanopowders
}

\author{
Isabel Santacruz $^{1,2^{*}}$, M. Isabel Nieto ${ }^{2}$, Jon Binner ${ }^{1}$, Rodrigo Moreno ${ }^{2}$ \\ ${ }^{1}$ IPTME, Loughborough University, Loughborough, LE11 3TU Leicestershire, UK \\ ${ }^{2}$ Instituto de Cerámica y Vidrio, CSIC, C/ Kelsen 5, Campus de Cantoblanco, 28049 \\ Madrid, Spain.
}

\begin{abstract}
Commercial nano $\mathrm{BaTiO}_{3}$ powders have been formed into green bodies using colloidal forming routes. A study of the rheological behaviour of the suspensions as a function of dispersant concentration and homogenisation time was made in order to prepare stable concentrated suspensions of the nanopowders. Bulk components were then manufactured using aqueous slip and gel casting involving polysaccharides that gel on cooling, i.e. agar. The performance of theses consolidation techniques for obtaining dense green bodies from the $\mathrm{BaTiO}_{3}$ nanopowders was studied. It was possible to prepare relatively big gel cast samples with a similar density and microstructure and in a shorter time compared to those obtained by slip casting.
\end{abstract}

Keywords: A. Suspensions; A. Shaping; B. Nano; D. BaTiO 3

\footnotetext{
${ }^{*}$ Corresponding author. Tel.: +34-91-735-5840; fax: +34-91-735-5843

E-mail address: cruz@icv.csic.es (I. Santacruz)

Currently at the Instituto de Cerámica y Vidrio (CSIC), 28049-Madrid, Spain
} 


\section{Introduction}

Nanostructured ceramics have the potential to display a broad range of unusual properties including superplasticity, transparency, enhanced mechanical properties and unusual dielectric properties, such as very high magnetoresistance and superparamagnetic properties. It is also possible to control band gaps in electronic materials. The real technological significance for nanoceramics is that these properties could lead to increased functionality, lower mass and miniaturised components as well as, in specific cases such as multilayer devices where the lower sintering temperature will allow the use of base metal technology, significant cost savings [1,2].

Unfortunately, there are some drawbacks to be overcome when producing nanostructured ceramics. Commercial nanopowders can now be produced in relatively large quantities, though to date they are generally strongly agglomerated. Various techniques are used in the literature to synthesise ceramic nanopowders including solid state reactions, hydrothermal routes, polymerization routes, sol-gel methods, microwave-assisted synthesis, and others [3-5]. Whilst each of them presents some advantages, each also has its limitations. Typical drawbacks of many of the methods of preparation are inhomogeneity, varied particle size distribution, poor reactivity and, in most cases where dry nanopowders are formed, the formation of hard agglomerates during synthesis.

Another difficulty found in nanoceramics manufacturing is the shaping of homogeneous green bodies. In general, colloidal processing allows the production of complex-shaped parts with a decreased number and size of pores and hence higher reliability ${ }^{6}$. In colloidal systems the interfaces play a key role in the surface properties, such as adsorption, surface charge, electric double layer, etc [6,7]. In the case of 
nanosized particles, their massive surface areas and hence substantial interaction forces between the particles make it more difficult to prepare stable nano-particle suspensions and study their colloidal properties.

Over the last few years, significant effort has been devoted to developing new forming methods for manufacturing complex-shaped ceramic products with minimal number of detrimental defects [8-10]. Slip casting is a well known technique for consolidating ceramic suspensions into complex shapes [11], although long times can be required, especially for nanosuspensions where the water filtration occurs with more difficulty. A further consequence is that the ultrafine porosity in the structure leads to severe difficulty in drying the parts without cracking. Gel casting is a bulk fabrication process for manufacturing near-net shaped components [12]. Thermogelling polysaccharides such as agar, agarose and carrageenan have demonstrated a high efficiency at low concentration when they are dissolved and incorporated into a hot slurry and cast or injected into a mould cavity where they cool to below their glass transition temperature, $\left.\left.\mathrm{T}_{\mathrm{g}}\right] 13,14\right]$. Gel casting with polysaccharides appears to be a good technique for consolidating ceramics with complex shapes and different sizes in a period that can be as short as a few seconds [15].

Barium titanate, $\mathrm{BT}\left(\mathrm{BaTiO}_{3}\right)$, is one of the most widely used ceramic raw materials in the electro-ceramic industry [16]. It is often processed via colloidal processing routes because it is extensively used in multilayer devices (e.g. multilayer ceramic capacitors, MLCC) where tape casting or screen printing are often used, where the thickness of each layer tends to be thinner. Due to the complexity of the system, most commercial applications are based on the use of organic BT suspensions [17], however, for environmental and economic reasons, there is a tendency to move towards 
the use of aqueous solvents. A number of studies have been performed focusing on the stability [18,19] and rheological behaviour [20] of aqueous BT suspensions. The ceramic is not thermodynamically stable in acidic suspensions because $\mathrm{Ba}^{2+}$ ions are leached out of the powder, which results in a titanium enriched surface layer. Ammonium polyacrylates have been found to be very useful in the stabilisation of submicron [21-23] and nanosized [20,24,25] BT suspensions at basic values of $\mathrm{pH}$, including the combination of an acrylic polyelectrolyte and a strong quaternary base such as TMAH $[20,26]$.

This work aims to study the preparation and rheological characterisation of concentrated aqueous suspensions of commercial nanosized powders of barium titanate (nanoBT) for shape forming by slip and gel casting, the latter using thermogelling polysaccharides. The performance of these consolidation techniques for obtaining dense green bodies from the BT nanopowder is discussed.

\section{Experimental}

The starting material was a commercial nanoBT powder (HPB-1000, TPL Inc., Albuquerque, USA) with a Ba/Ti molar ratio of 0.996-1.004. The surface area was measured by one point nitrogen adsorption (Monosorb, Quantachrome, Florida, USA), and the density by He pycnometry (Quantachrome, Florida, USA). The particle size was calculated from the BET area data and checked by field emission gun scanning electron microscopy, FEG-SEM (Leo 1530 VP, Elektronenmikroskopie GmbH, Oberkochen, Germany) and transmission electron microscopy, TEM, (JEOL 2000FX, Jeol, Tokyo, Japan). Characterisation was completed with thermogravimetric analysis, TGA (STA 
409, Netzsch, Bavaria, Germany) and X-Ray Diffraction (D5000, Siemens, Nuremberg, Germany).

Aqueous suspensions were prepared based on earlier work published elsewhere [27]. Essentially, it involved adding tetramethylammonium hydroxide, TMAH (supplied by Aldrich-Chemie, Sant Louis, USA, as a $25 \mathrm{wt} \%$ aqueous solution) to deionised water to generate a $\mathrm{pH}$ value of 11.0 ; this involved mixing $0.12 \mathrm{~g}$ of the TMAH solution into $280.0 \mathrm{~g}$ of water. Then, $1.0-3.0 \mathrm{wt} \%$ (in terms of the active matter and on a dry solids basis) of an ammonium salt of polyacrylic acid (Duramax D3005, Rohm \& Haas, USA) was added as a deflocculant to the solution $[20,27]$ prior to the addition of sufficient of the nanoBT powder to generate suspensions with solids loadings of 70,75 and $80 \mathrm{wt} \%$ (28, 33 and $40 \mathrm{vol} \%$, respectively). The suspensions were mixed using by mechanical agitation with helices and further homogenised using ultrasound (a $400 \mathrm{~W}$ sonication probe, UP400S, Hielscher Ultrasonics GmbH, Stuttgart, Germany) for up to $10 \mathrm{~min}$. Suspensions were cooled in an ice-water bath during ultrasonication in order to avoid heating.

A commercially available agar (Grand Agar, Hispanagar S.A., Burgos, Spain) was used as the gelling agent. Solutions of agar were prepared at concentrations of $6 \mathrm{wt} \%$ by heating in a pressure vessel $[14,15]$ in order to achieve the $110^{\circ} \mathrm{C}$ required for its full dissolution without the water boiling.

The rheological behaviour of the suspensions was studied using a rheometer (Haake RS50, Karlsruhe, Germany) with a double-cone and plate measuring system, provided with a solvent trap to reduce evaporation. Flow curves were obtained with a three-stage measuring program with a linear increase of shear rate from 0 to $1000 \mathrm{~s}^{-1}$ in $300 \mathrm{~s}$, a plateau at $1000 \mathrm{~s}^{-1}$ for $120 \mathrm{~s}$, and a further decrease to zero shear rate in $300 \mathrm{~s}$ 
(Controlled Rate mode, CR). The rheological behaviour was studied for both fresh slips and for slips aged in a shaker (KS 260 basic, IKA, Staufen, Germany) for $20 \mathrm{~h}$. The evolution of the viscosity with temperature was measured at a shear rate of $100 \mathrm{~s}^{-1}$ by heating from 25 to $70^{\circ} \mathrm{C}$ at a heating rate of $2.1^{\circ} \mathrm{C} / \mathrm{min}$ and then cooling from 60 to $25^{\circ} \mathrm{C}$ at a cooling rate of $1.2^{\circ} \mathrm{C} / \mathrm{min}$.

The influence of the volume fraction of solids, the dispersant concentration, the time of ultrasonic mixing and the effect of ageing on the suspension rheology was studied. Values of viscosity extrapolated to infinite shear rates were calculated using the Cross model on flow curves measured using the Controlled Rate mode. The maximum packing fraction of the suspensions was estimated by using the Krieger-Dougherty model with the calculated limit viscosities.

BT green bodies were primarily obtained by gel casting, although slip casting on plaster moulds was also carried out for comparative purposes. In the former, bars with dimensions of $60 \mathrm{~mm} \times 10 \mathrm{~mm} \times 10 \mathrm{~mm}$ were consolidated in less than $1 \mathrm{~min}$, and in the latter, green bodies were cast into discs measuring $9 \mathrm{~mm}$ diameter by $10 \mathrm{~mm}$ thick. To prepare the gel casting suspensions, the well-dispersed $80 \mathrm{wt} \%$ solids content BT nanosuspensions were heated to a temperature higher than the agar gelation temperature, viz. $\sim 40^{\circ} \mathrm{C}$ for the $6 \mathrm{wt} \%$ solution $[14,15]$. The agar solution was then added to the nanosuspension at a concentration of $0.5 \mathrm{wt} \%$ with respect to the dry solids content whilst maintaining a temperature of $>50^{\circ} \mathrm{C}$. The suspension was subsequently poured into the steel mould and then allowed to cool. All samples were dried under lab. conditions. The green densities of the resulting bodies were measured via the Archimedes' method using mercury and the green fracture surfaces were observed by using scanning electron microscopy, SEM (Zeiss DSM40, Oberkochen, Germany). 


\section{Results and Discussion}

Fig. 1 shows the nanostructure of the starting powder as observed by FEG-SEM and TEM. The powder consisted of hard spherical granules with diameters ranging from 5 to $40 \mu \mathrm{m}$, typical of a spray dried powder, fig. 1. Higher magnification pictures revealed that particles had uniform shape. The measured density was $5.5 \mathrm{~g} \mathrm{~cm}^{-3}$, lower than the crystallographic density of the cubic phase of $\mathrm{BaTiO}_{3}$, which was the only one detected by XRD analysis. The surface area measured by the BET method was $17.0 \mathrm{~m}^{2}$

$\mathrm{g}^{-1}$, leading to a calculated average particle size, $\mathrm{d}_{\mathrm{BET}}$, of $64 \mathrm{~nm}$ in line with the electron microscopy results. The weight loss of the powder after heating at $600^{\circ} \mathrm{C}$ was $\sim 0.7 \%$ indicating that the powders contained relatively little by way of organic additives, so that no calcination step was needed for further processing in contrast to that observed for another commercial BT nanopowder [27].

Fig. 2 shows the flow behaviour for a $70 \mathrm{wt} \% \mathrm{BT}$ suspension prepared using 0 to $3 \mathrm{~min}$ of ultrasonication, stabilization being provided with $\mathrm{TMAH}$ and $2.0 \mathrm{wt} \%$ of polyelectrolyte in all cases. Suspensions with insufficient ultrasound homogenisation, i.e. $<1 \mathrm{~min}$, displayed a very high viscosity and thixotropy, both of which were significantly reduced with increasing exposure to the ultrasound. A comparison of fresh and aged suspensions prepared with an ultrasonication time of 3 min demonstrated that the suspension prepared with this degree of homogenisation was very stable for at least 20 hours. This degree of stability is very high when compared with other nano ceramic suspensions [28]. 
For the $75 \mathrm{wt} \%$ solids loading suspensions, a longer ultrasonication time range, from 0 to 6 min, was studied. Fig. 3 shows the flow curves of the fresh suspensions as well as the ageing effect on the suspension exposed to ultrasound for $6 \mathrm{~min}$. The latter can be seen to remain the same as that for the freshly prepared suspension indicating that the suspensions not only have a low viscosity but are also stable with time.

From the above results, it was confirmed that longer ultrasonication times were required for higher solids loadings, as expected. For this reason, ultrasonication times from 0 to $10 \mathrm{~min}$ were studied for the $80 \mathrm{wt} \%$ solids loading suspensions. As observed in the corresponding flow curves, fig. 4 , the viscosity of the suspensions decreased with increasing ultrasonication time up to $10 \mathrm{~min}$, the limit investigated. After ageing for 20 $\mathrm{h}$, the viscosity had decreased very slightly but otherwise there was very little change, again confirming the stability of the nanosuspension.

All the suspensions discussed above were prepared with the same dispersant content, TMAH and $2.0 \mathrm{wt} \%$ D3005 in line with previous work [27]. However, as indicated in the experimental section, $80 \mathrm{wt} \%$ suspensions were also prepared with 1 and $3 \mathrm{wt} \%$ D3005 in order to establish the most adequate dispersing conditions. Table I shows the viscosity values for these suspensions, taken at $100 \mathrm{~s}^{-1}$ in the ascending ramp of their viscosity curves. This table confirms that viscosity was always lowest for a D3005 dispersant content of $2 \mathrm{wt} \%$ for any ultrasound homogenisation times.

In order to study the influence of the solids loading on the viscosity of the suspensions with $2.0 \mathrm{wt} \%$ dispersant and to calculate the maximum packing fraction, the experimental data were fitted to the Krieger-Dougherty model (eq. 1) where the experimental viscosity values were obtained by extrapolation of viscosity to infinite shear rate $\left(\eta_{\infty}\right)$ for each suspension according to the Cross model. 
$\eta=\eta_{s}\left(1-\phi / \phi_{m}\right)^{-[\eta] \phi_{m}}$

where $\eta_{\mathrm{s}}$ is the solvent viscosity, $\phi$ the volume fraction, $\phi_{\mathrm{m}}$ the maximum packing, [ $\left.\eta\right]$ the intrinsic viscosity. Since $[\eta]$ is very difficult to obtain by experimental routes, in order to predict the evolution of viscosity with solids loading in suspensions with nonspherical particles, the modified Krieger-Dougherty model substitutes the factor $[\eta] \phi_{\mathrm{m}}$ by a factor $\mathrm{n}$, which can be fitted by a large variety of suspensions of different materials. [29] Table II reports the limit viscosities of suspensions with different solids fractions prepared at different ultrasound times. Fig. 5 shows the $\eta_{\mathrm{r}}-\phi$ curves of the ultrasonicated suspensions calculated in the high shear rate region by fitting the experimental $\eta_{\infty}$ data (symbols) to the Krieger-Dougherty model (continuous lines). Table III shows the $\mathrm{n}$ and $\phi_{\max }$ values obtained for the suspensions after 0,2 and 6 min of ultrasonication, using the theoretical model. In all cases, a good approximation has been found within the data range examined, fig. 5, although in the case where no ultrasound was used, the approximation is not as good as for the other two curves since the suspensions were not as stable when ultrasound was not used to homogenise them. These results agree with the higher $\mathrm{n}$ value and lower $\phi_{\max }$ obtained when they are compared with the 2 and 6 min ultrasound suspensions, where $\phi_{\max }$ values up to 0.54 were found for 6 min of ultrasound and a value of 2.5 for $n$ was obtained in both cases. This demonstrates that ultrasonication allows homogeneous suspensions to be obtained whose packing behaviour can be accurately predicted from rheological measurements. Therefore, it can be deduced that, within the range of study, the longer the ultrasonication time the lower the viscosity values attained. This means that more 
homogeneous suspensions with higher maximum packing factors (i.e., green densities) can be obtained.

The nano-particulate slip cast samples required long times for the filtration of the suspension water through the plaster mould (viz. $4 \mathrm{~mm}$ thickness required more than 1 hour vs. 4 min for obtaining similar thickness when submicron suspensions [30] are slip cast). The longer times needed when bigger size samples were cast would lead to defects. For this reason, it is believed that the manufacture of net-shaped bulk bodies from nanosized powders needs alternative forming routes for obtaining homogeneous, large samples in a reasonable time. In this sense, the authors believed that thermogelation with agar was one of the best options to study as an alternative to slip casting and the current work sought to evaluate the rheological behaviour on cooling to evaluate the gel formation.

Figure 6 shows the evolution of the viscosity during cooling of the agar solution; there was a sharp increase in the viscosity at $-38^{\circ} \mathrm{C}$ due to gelation. The evolution of viscosity of the $80 \mathrm{wt} \%$ BT nanosuspension on heating is also shown and it is observed that the suspension destabilised at temperatures above $\sim 60-65^{\circ} \mathrm{C}$, this being the upper temperature limit for this process. For this reason, suspensions were heated to $50^{\circ} \mathrm{C}$ prior to the addition of the agar solution. The nanosuspension / polysaccharide mixtures were poured into steel moulds to promote the gelation on cooling, which was immediate. The increase of $\eta$ on gelation could not be measured because the torque of the rheometer was exceeded.

The green slip cast samples had densities of $\sim 58 \%$ compared to the measured powder density, or $54 \%$ of the theoretical crystallographic density of cubic BT. The equivalent density values for the gel cast samples were $57 \%$ and $52 \%$ respectively, very 
similar to those for the slip cast bodies. Figures $7 \mathrm{a}$ and $7 \mathrm{~b}$ show the SEM

microstructures of the green samples obtained by slip and gel casting respectively. The agglomerates observed in the as-received powder may still be seen in both bodies.

Previous studies have demonstrated that these spray dried agglomerates also persist in dry pressed samples produced using $500 \mathrm{MPa}$ of applied pressure after micronising the powder for several minutes [31]. In that case, the green density of the bodies formed was lower, just $53 \%$ of the measured powder density and $48 \%$ of the crystallographic density.

It is important to note that gel casting leads to samples with a similar microstructure than slip cast bodies and no additional agglomerates are formed due to the polysaccharide addition or any other step of this shaping route. Hence, gel casting with agar is a powerful forming technique to produce net-shaped bodies in a very short time (consolidation takes less than $1 \mathrm{~min}$ ) and with similar density values than those obtained by slip casting of nanosuspensions. Then, depending on the size and shape of the required nanostructured samples, it is possible to select slip or gel casting with polysaccharides as forming process.

\section{Conclusions}

Stable nano- $\mathrm{BaTiO}_{3}$ aqueous suspensions were prepared with solids loadings as high as $80 \mathrm{wt} \%$ by dispersing with $2 \mathrm{wt} \%$ ammonium polyelectrolyte in combination with TMAH and using an ultrasound probe for homogenisation times of up to $10 \mathrm{~min}$. Green bodies of $\mathrm{BaTiO}_{3}$ were prepared by both slip and gel casting, both showing similar characteristics of density and microstructure. Unbroken agglomerates, similar to those observed in the as-received powder, were present in the green samples. The 
densities of the green samples prepared by slip and gel casting were 58 and $57 \%$, respectively, compared to the measured density of the as-received powder; higher than that obtained by die pressing in a related study.

Under the best conditions determined in the work, the maximum packing fraction obtained by fitting the experimental limit viscosities to the modified KriegerDougherty model was 0.54 , slightly lower than the green densities obtained experimentally.

\section{Acknowledgments}

This work has been partially supported by Spanish Ministry of Education and Science (CICYT projects MAT2006-13480 and MAT2006-01038). Dr. Santacruz thanks ESF and CSIC for the concession of a postdoctoral contract I3P-PC2005L. 


\section{References}

1. M. Mayo, Processing of nanocrystalline ceramics from ultrafine particles, Int. Mater. Rev., 41 (85) (1996) 1743-2804.

2. J.R. Groza, Sintering of nanocrystalline powders, Int. J. Powder Met., 35 (7) (1999) 59-66.

3. C. Pitha, D. Hennings, R. Waser, Progress in the Synthesis of Nanocrystalline $\mathrm{BaTiO}_{3}$ Powders for MLCC, Int. J. Appl. Ceram. Tech., 2 (1) (2005) 1-14.

4. C. Laberty-Robert, F. Ansart, S. Castillo, G. Richard, Synthesis of YSZ powders by the sol-gel method: surfactant effects on the morphology, Solid State Sciences, 4 (8) (2002) 1053-59.

5. L. Combemale, G. Caboche, D. Stuerga, D. Chaumont, Microwave synthesis of yttria stabilized zirconia, Mater. Res. Bull., 40 (3) (2005) 529-36.

6. F.F. Lange, Powder processing science and technology for increased reliability, J. Am. Ceram. Soc., 72 (1) (1989) 3-15.

7. J.N. Israelachvili, Intermolecular and Surface Forces, 2nd ed., Academic Press, N.Y., 1992.

8. L.A. Wang, F. Aldinger, Near-net shape forming of advanced ceramics, Adv. Eng. Mater., 3 (2) (2000) 110-13.

9. W.M. Sigmund, N.S. Bell, L. Bergström, Novel powder-processing methods for advanced ceramics, J. Am. Ceram. Soc., 83 (7) (2000) 1557-74.

10. J.A. Lewis, Colloidal processing of ceramics, J. Am. Ceram. Soc., 83 (10) (2000) 2341-59. 
11. F. Boschini, B. Guillaume, A. Rulmont, R. Cloots, R. Moreno, Slip casting of barium zirconate aqueous concentrated suspensions, J. Eur. Ceram. Soc., 26 (2006) 1591-98.

12. O.O. Omatete, M.A. Janney, S.D. Nunn, Gel casting: from laboratory development toward industrial production, J. Eur. Ceram. Soc., 17 (1997) 407-13.

13. A.J. Fanelli, R.D. Silvers, W.S. Frei, J.V. Burlew, G.B. Marsh, New Aqueous Injection Molding Process for Ceramic Powders, J. Am. Ceram. Soc., 72 (10) (1989) 1833-36.

14. I. Santacruz, M.I. Nieto, R. Moreno, P. Ferrandino, A. Salomoni, I. Stamenkovic, Aqueous injection moulding of porcelains, J. Eur. Ceram. Soc., 23 (2003) 2053-60. 15. I. Santacruz, M.I. Nieto, R. Moreno, Alumina bodies with near-to-theoretical density by aqueous gelcasting using concentrated agarose solutions, Ceram. Int., 31 (2005) 439-45.

16. D.H. Yoon, B.I. Lee, $\mathrm{BaTiO}_{3}$ properties and powder characteristics for ceramic capacitors, J. Ceram. Process. Res, 3 (2) (2002) 41-47.

17. L. Bergström, K. Shinozaki, H. Tomiyama, N. Mizutani, Colloidal processing of a very fine $\mathrm{BaTiO}_{3}$ powder - Effect of particle interactions on the suspensions properties, consolidation, and sintering behaviour, J. Am. Ceram. Soc., 80 (2) (1997) 291-300. 18. C.W. Chiang, J.H. Jean, Effects of barium dissolution on dispersing aqueous barium titanate suspensions, Mater. Chem. Physics, 80 (3) (2003) 647-55.

19. J.H. Hean, H.R. Wang, Effects of solids loading, $\mathrm{pH}$, and polyelectrolyte addition on the stabilization of concentrated aqueous $\mathrm{BaTiO}_{3}$ suspensions, J. Am. Ceram. Soc., 83 (2) (2000) 277-80. 
20. I. Santacruz, M.I. Nieto, J. Binner, R. Moreno R., Dispersion and rheology of aqueous suspensions of nanosized $\mathrm{BaTiO}_{3}$, Submitted to J. Am. Ceram. Soc., 2007. 21. Z.C. Chen, T.A. Ring, J. Lemaitre, Stabilization and processing of aqueous $\mathrm{BaTiO}_{3}$ suspension with polyacrylic acid, J. Am. Ceram. Soc., 75 (12) (1992) 3201-208. 22. C. Gomez-Yagnez, H. Balmori-Ramirez, F. Martinez, Colloidal processing of $\mathrm{BaTiO}_{3}$ using ammonium polyacrylate as dispersant, Ceram. Inter., 26 (6) (2000) 60916.

23. W.J. Tseng, S.Y. Li, Rheology of colloidal $\mathrm{BaTiO}_{3}$ suspension with ammonium polyacrylate as dispersant, Mater. Sci. Eng. A, 333 (1-2) (2002) 314-19.

24. Z.G. Shen, J.F. Chen, H.K. Zou, J. Yun, Dispersion of nanosized aqueous suspension of barium titanate with ammonium polyacrylate, J. Colloid Interface Sci., 275 (1) (2004) 158-64.

25. Z.G. Shen, J.F. Chen, H.K. Zou, J. Yun, Rheology of colloidal nanosized $\mathrm{BaTiO}_{3}$ suspension with ammonium polyacrylate as dispersant, Colloids and Surfaces A: Physicochem. Eng. Aspects, 244 (1-3) (2004) 61-66.

26. F. Boschini, A. Rulmont, R. Cloots, R. Moreno, Colloidal stability of aqueous suspensions of barium zirconate, J. Eur. Ceram. Soc., 25 (2005) 3195-201.

27. I. Santacruz, M.I. Nieto, J. Binner, R. Moreno, Wet forming of concentrated nano $\mathrm{BaTiO}_{3}$ suspensions, J. Eur. Ceram. Soc., In Press

28. O. Burgos-Montes, M.I. Nieto, R. Moreno, Mullite compacts obtained by colloidal filtration of alumina powders dispersed in colloidal silica suspensions, Ceram. Int., 33 (2007) 327-32.

29. C.A. Gutiérrez, R. Moreno, Interparticle Potentials in Nonaqueous Silicon Nitride Suspensions, J. Am. Ceram. Soc., 86 (1) (2003) 59-64. 
30. R. Moreno, J.S. Moya, J. Requena, Slip Casting of Zircon by Using an Organic Surfactant, Ceram. Int., 17 (1991) 17 37-40.

31. I. Santacruz, C. Wells, J. Binner, Microwave Two Stage Sintering of Nanostructured Barium Titanate, Proceedings of the $10^{\text {th }}$ International Conference on Microwave and High Frequency Heating, 10-Ampere, Italy, 2005. 


\section{Captions}

Fig. 1. (a) and (b) FEG-SEM and (c) TEM micrographs of the as-received powder. Fig. 2. Flow curves of (a) fresh and (b) $20 \mathrm{~h}$ aged $70 \mathrm{wt} \%$ suspensions with different ultrasonication times.

Fig. 3. Flow curves of $75 \mathrm{wt} \%$ suspensions with different ultrasonication times.

Fig. 4. Flow curves of $80 \mathrm{wt} \%$ suspensions with different ultrasonication times.

Fig. 5. Comparison of experimental and calculated (Krieger-Dougherty model) data for the variation of the viscosity with the volume fraction of solids as a function of ultrasound time.

Fig. 6. Evolution of viscosity with temperature for the agar solutions on cooling and the $80 \mathrm{wt} \%$ BT suspensions on heating, both at a shear rate of $100 \mathrm{~s}^{-1}$.

Fig. 7. SEM micrographs of the green samples prepared by (a) slip casting and (b) gel casting from $80 \mathrm{wt} \%$ nanosuspensions prepared using $10 \mathrm{~min}$ of ultrasound.

Table I. Experimental viscosity values for $80 \mathrm{wt} \%$ suspensions with TMAH and 1, 2 and $3 \mathrm{wt} \% \mathrm{D} 3005$ taken at $100 \mathrm{~s}^{-1}$ in the ascending ramp of their viscosity curves Table II. Extrapolated values of the viscosity for $80 \mathrm{wt} \%$ BT nanosuspensions as a function of ultrasound time.

Table III. Calculated $\mathrm{n}$ and $\phi_{\max }$ values for the suspensions in Table II. 


\begin{tabular}{ccc}
\hline D3005/ wt \% & Ultrasonication / min & Viscosity / mPa s \\
\hline 1 & 0 & 2090 \\
\hline \multirow{2}{*}{2} & 0 & 1500 \\
& 4 & 279 \\
& 8 & 206 \\
\hline \multirow{2}{*}{3} & 0 & 1770 \\
& 4 & 700 \\
& 8 & 670 \\
\hline
\end{tabular}

Table I. Experimental viscosity values for $80 \mathrm{wt} \%$ suspensions with TMAH and 1, 2 and $3 \mathrm{wt} \% \mathrm{D} 3005$ taken at $100 \mathrm{~s}^{-1}$ in the ascending ramp of their viscosity curves 


\begin{tabular}{ccc}
\hline Ultrasound / $\mathbf{m i n}$ & $\phi$ & $\eta_{\infty} / \mathbf{m P a} \mathbf{~}$ \\
\hline & 0.30 & 31.8 \\
0 & 0.35 & 148.6 \\
& 0.42 & 563.0 \\
\hline \multirow{2}{*}{2} & 0.30 & 6.0 \\
& 0.35 & 15.3 \\
& 0.42 & 84.0 \\
\hline \multirow{2}{*}{6} & 0.30 & 4.5 \\
& 0.35 & 9.3 \\
& 0.42 & 29.9 \\
\hline
\end{tabular}

Table II. Extrapolated values of viscosity of optimised $\mathrm{BaTiO}_{3}$ suspensions 


\begin{tabular}{ccc} 
Ultrasound / $\mathbf{m i n}$ & $\mathbf{n}$ & $\phi_{\max }$ \\
\hline 0 & 3.5 & 0.49 \\
2 & 2.5 & 0.51 \\
6 & 2.5 & 0.54 \\
\hline
\end{tabular}

Table III. Values of $\mathrm{n}$ and $\phi_{\max }$ obtained for the suspensions after 0,2 and $6 \mathrm{~min}$ sonication after fitting to the Krieger-Dougherty model. 

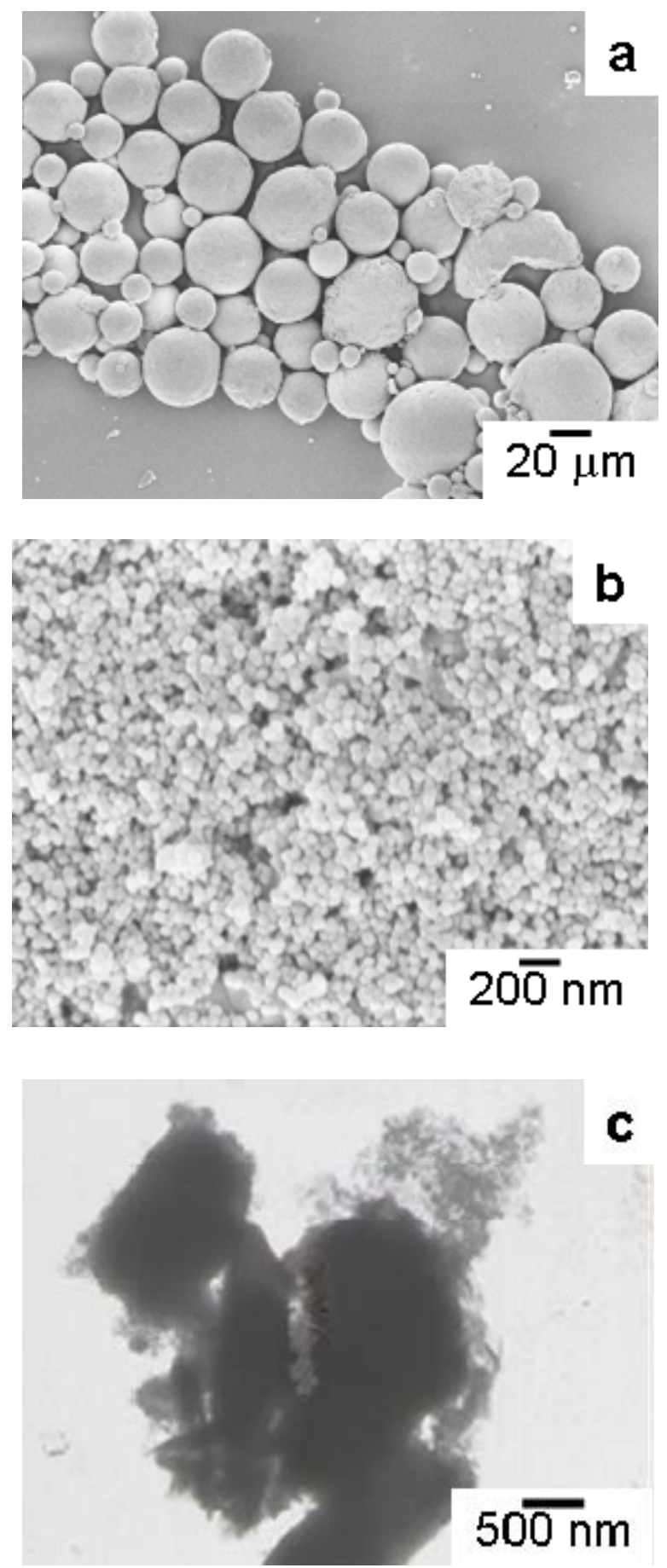

Fig. 1 

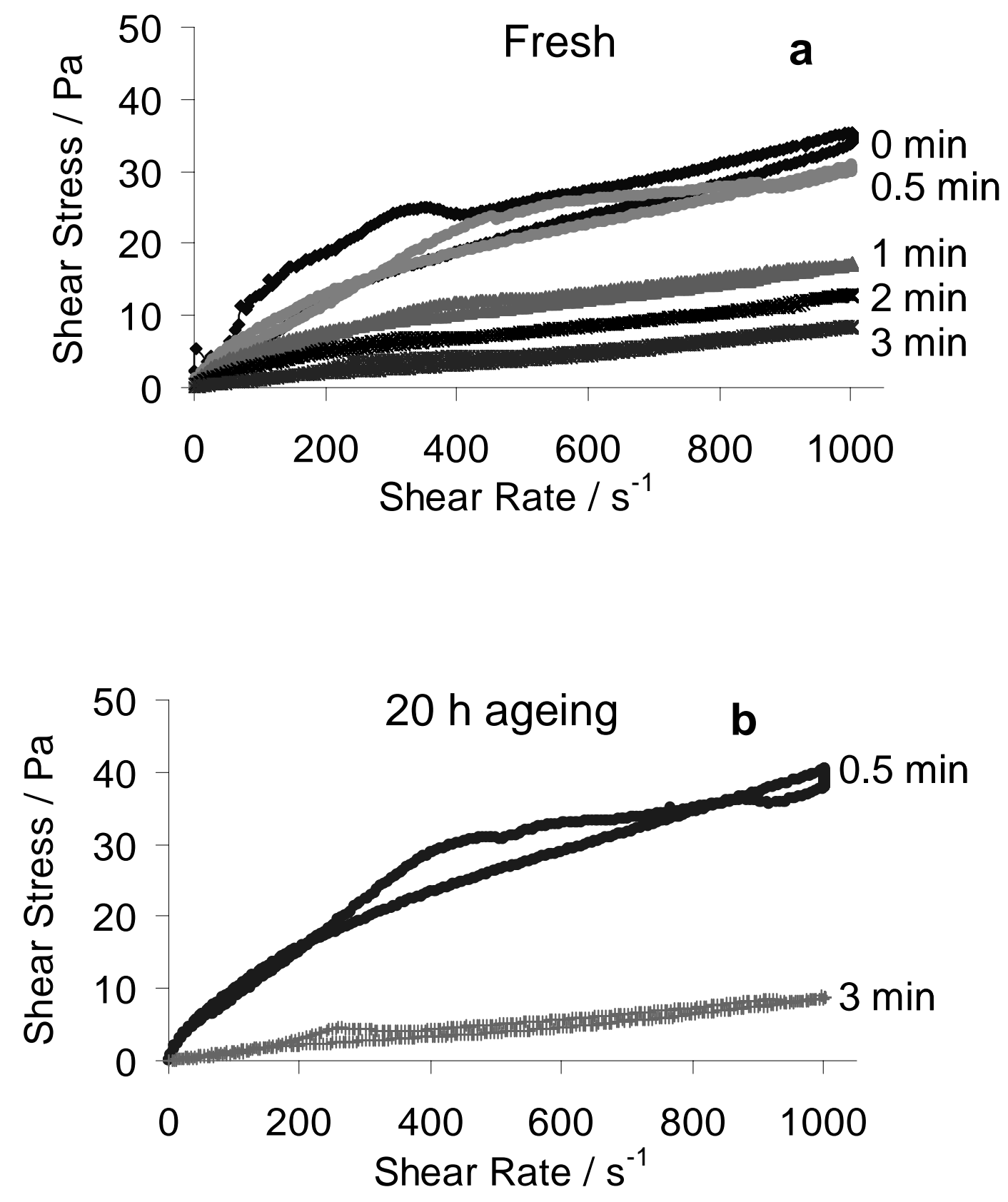

Fig. 2 


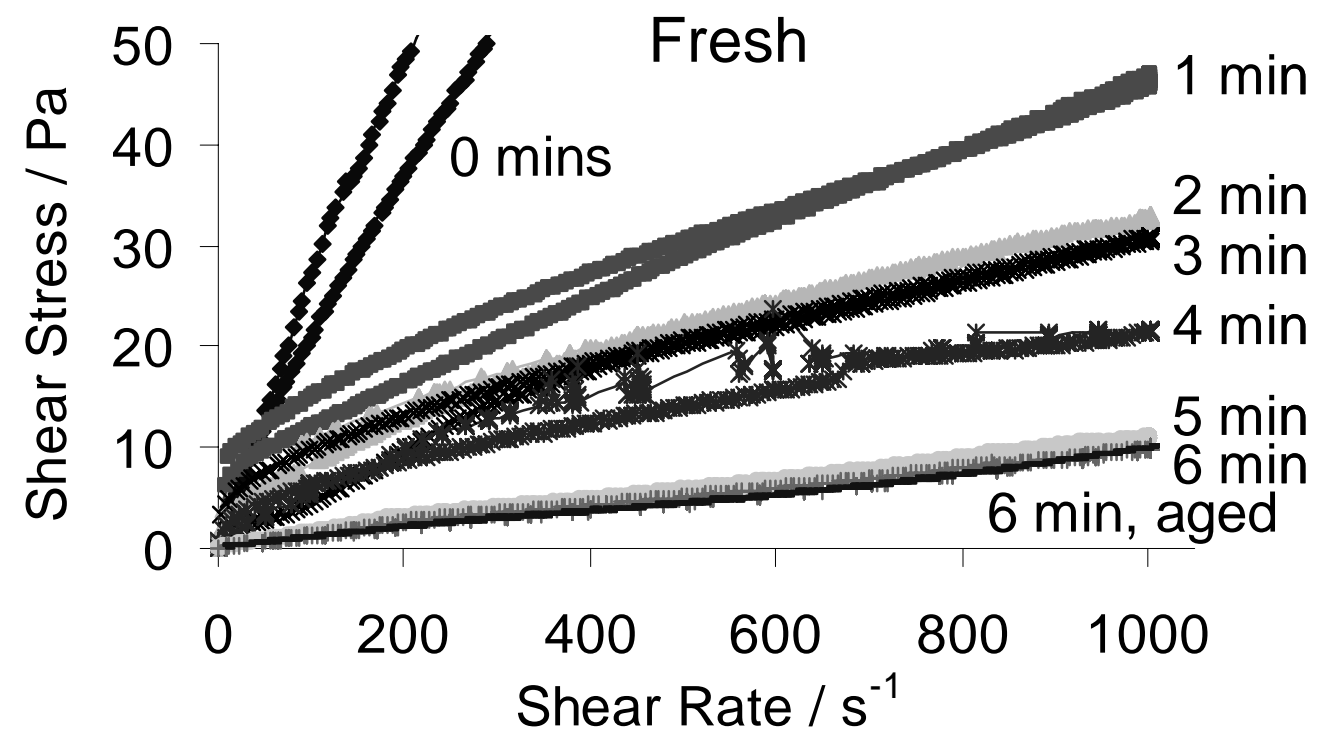

Fig. 3 


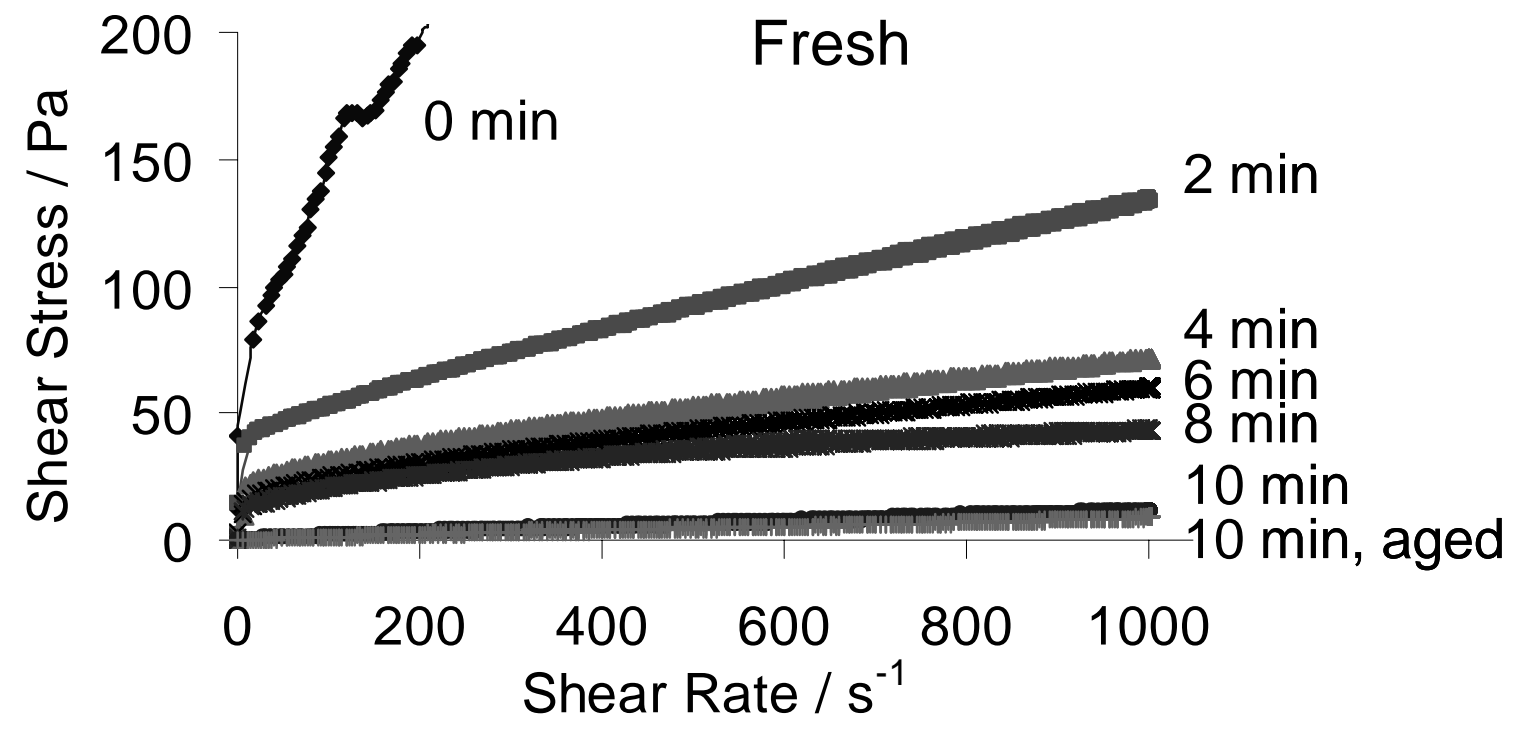

Fig. 4 


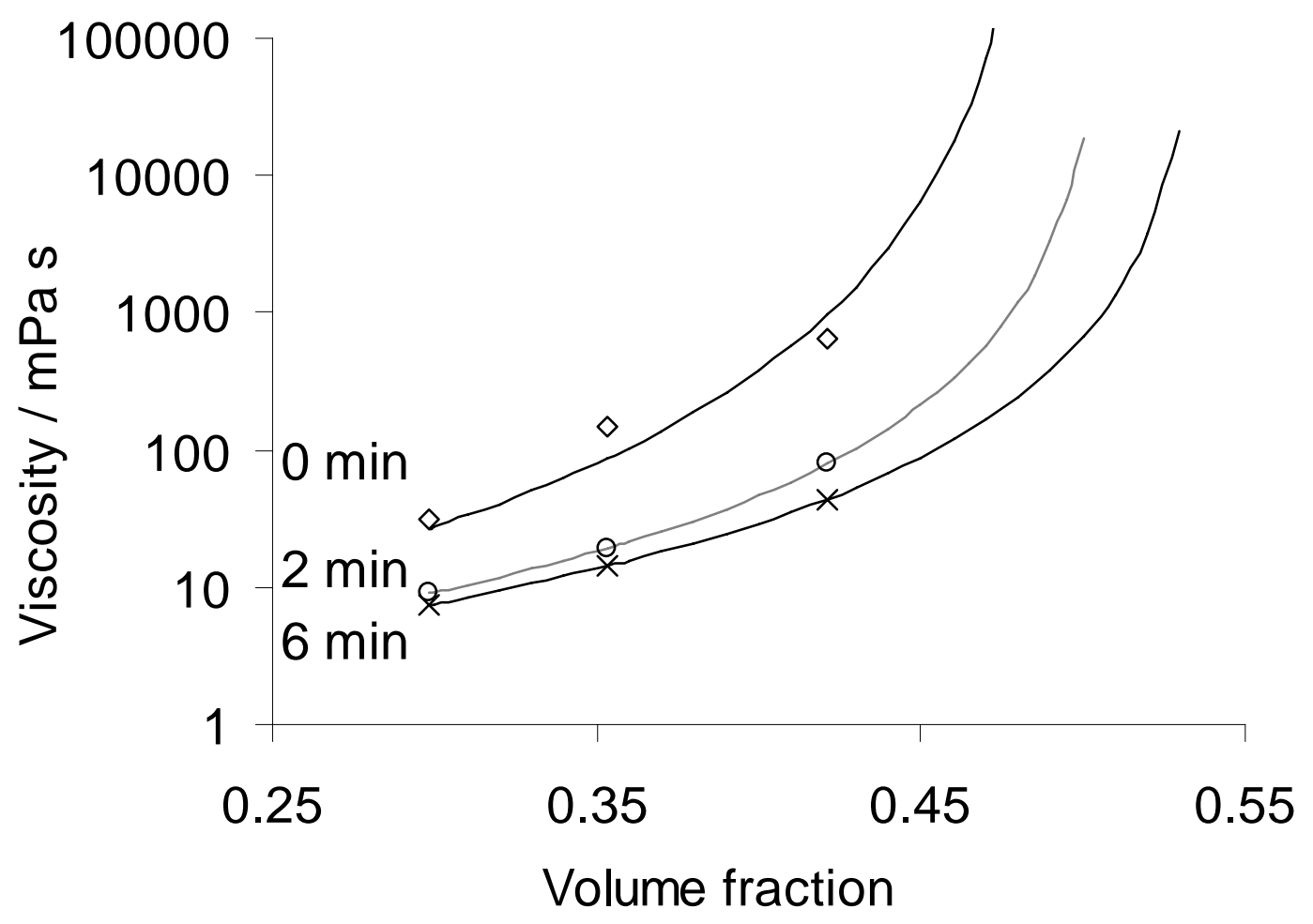

Fig. 5 


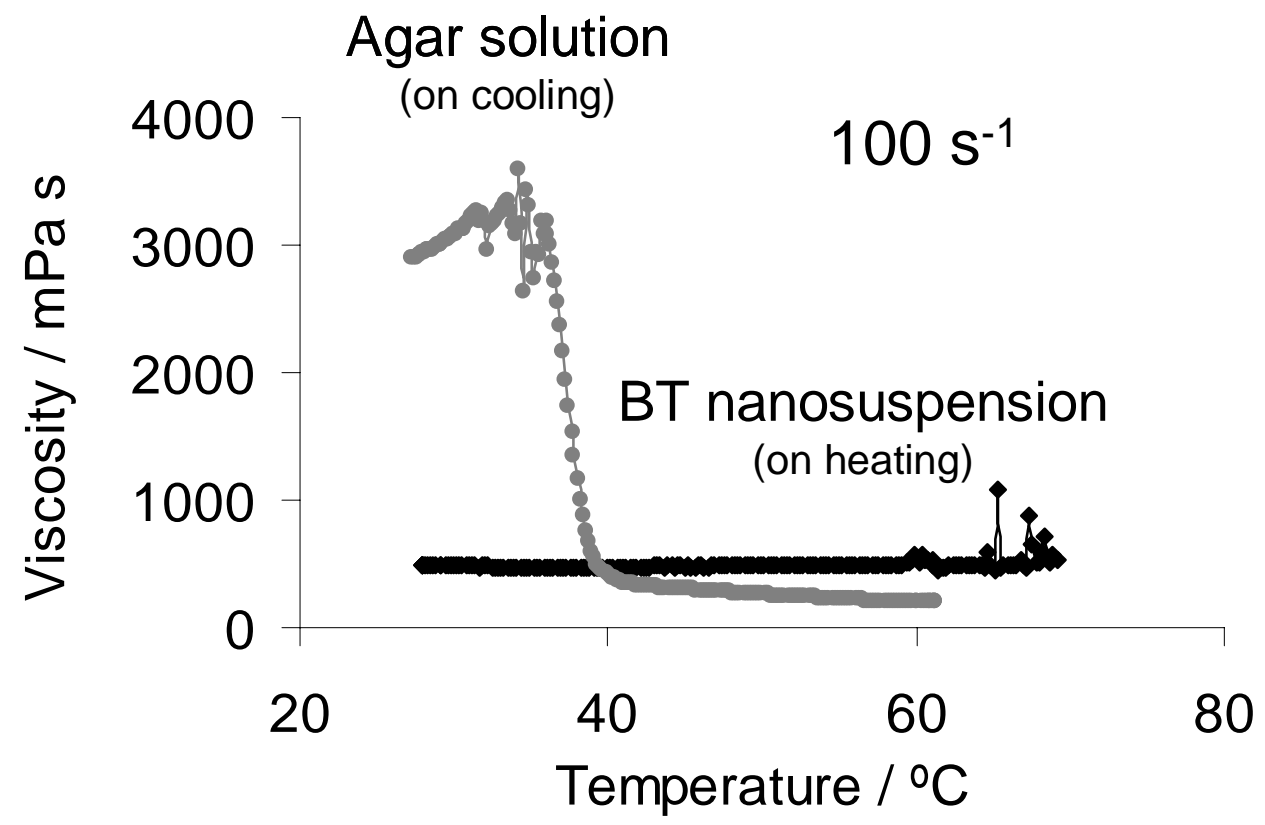

Fig. 6 

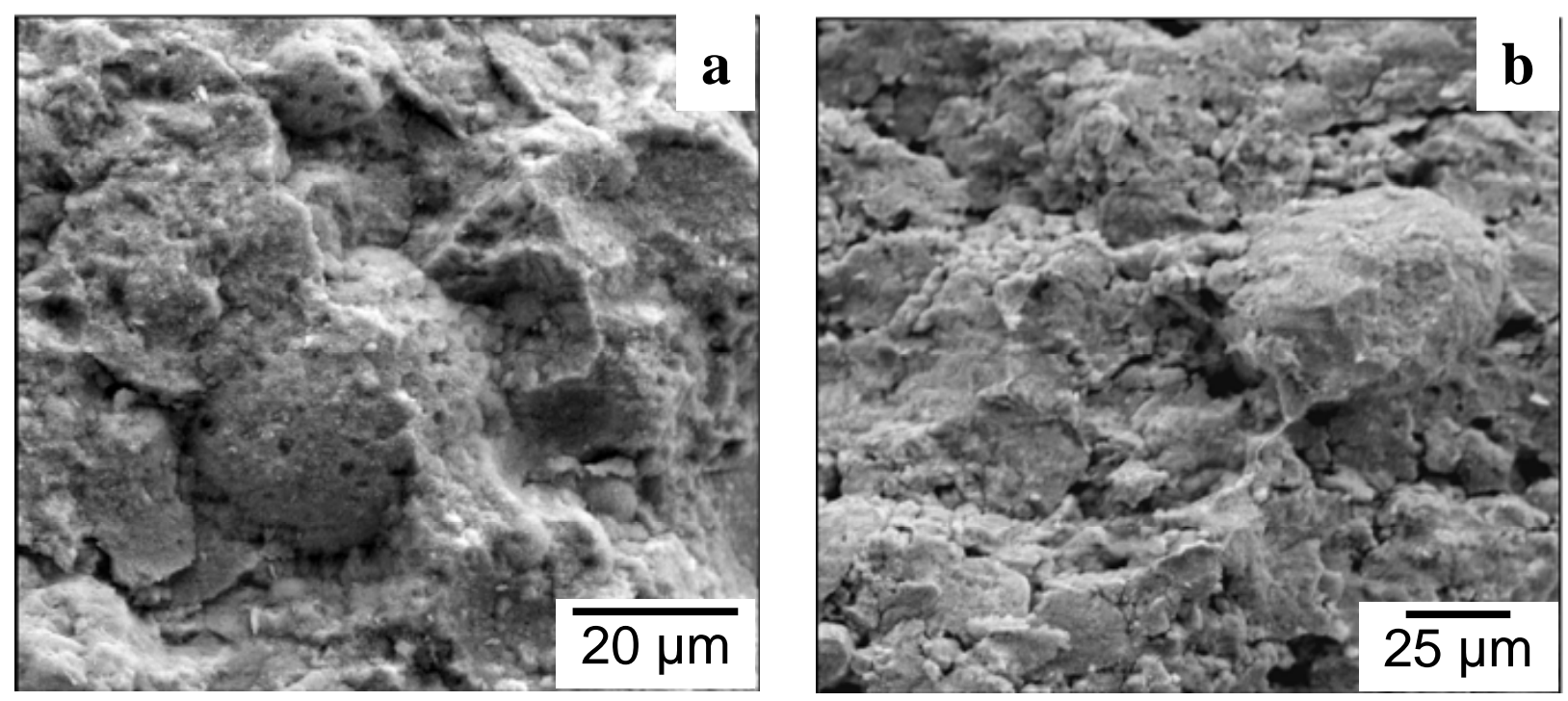

Fig. 7 